\title{
Erratum to: The impact of common co-morbidities (as measured using the Charlson index) on hip fracture risk in elderly men: a population-based cohort study
}

\author{
C. Reyes • P. Estrada $\cdot$ X. Nogués $\cdot$ P. Orozco $\cdot$ C. Cooper $\cdot$ \\ A. Díez-Pérez • F. Formiga • J. González-Macías • D. Prieto-Alhambra
}

Published online: 17 April 2014

(C) International Osteoporosis Foundation and National Osteoporosis Foundation 2014

Erratum to: Osteoporos Int

DOI 10.1007/s00198-014-2682-9

The name of the eighth author was rendered wrongly; the correct name is J. González-Macías.

The online version of the original article can be found at http://dx.doi.org/ 10.1007/s00198-014-2682-9.

\section{Reyes}

Primary Health Care Center, Eap Sardenya-Biomedical Resarch Institute Sant Pau (IIB Sant Pau), c/Sardenya 466, Barcelona, Spain

C. Reyes $\cdot$ P. Estrada $\cdot$ P. Orozco $\cdot$ D. Prieto-Alhambra GREMPAL Research Group, IDIAP Jordi Gol Primary Care Research Institute, Universitat Autònoma de Barcelona, Barcelona, Spain

P. Estrada $\cdot$ P. Orozco

Primary Health Care Gotic, Institut Catala de la Salut, Universitat de Barcelona, Barcelona, Spain

X. Nogués · A. Díez-Pérez • D. Prieto-Alhambra

Unitat de Recerca en Fisiopatologia Òssia i Articular (URFOA) and RETICEF, IMIM Research Foundation, Parc de Salut Mar and Instituto de Salud Carlos III, Barcelona, Spain
C. Cooper · D. Prieto-Alhambra

MRC Lifecourse Epidemiology Unit, University of Southampton,

Southampton, United Kingdom

C. Cooper $\cdot$ D. Prieto-Alhambra $(\bowtie)$

Oxford NIHR Musculoskeletal Biomedical Research Unit, Nuffield Department of Orthopaedics, Rheumatology and Musculoskeletal Sciences, University of Oxford, Windmill Road, OX3 7LD Oxford, United Kingdom

e-mail: Daniel.prietoalhambra@ndorms.ox.ac.uk

F. Formiga

Geriatric Unit, Internal Medicine Service, Hospital Universitari de Bellvitge, IDIBELL, Barcelona, Spain

J. González-Macías

Department of Internal Medicine, Hospital U.M. Valdecilla-IFIMAV, RETICEF, Santander, Spain 